\title{
Measuring the surface magnetic fields of magnetic stars with unresolved Zeeman splitting ${ }^{\star}$
}

\author{
J. D. Bailey ${ }^{1,2}$ \\ ${ }^{1}$ Max-Planck-Institut für extraterrestrische Physik, Giessenbachstrasse 1, 85748 Garching, Germany \\ e-mail: jeffbailey@mpe.mpg.de \\ 2 School of Physics and Astronomy, University of Leeds, Leeds, LS2 9JT, UK \\ Received 8 April 2014 / Accepted 7 July 2014
}

\section{ABSTRACT}

\begin{abstract}
Aims. High-dispersion, archival spectra of magnetic Ap stars with resolved Zeeman components in Stokes $I$ are used to derive a simple relation that can be utilised to estimate the mean surface field strengths of stars with $v \sin i \gtrsim 10 \mathrm{~km} \mathrm{~s}^{-1}$.

Methods. For each star, the mean surface field, as measured from the observed splitting in Fe II at $6149 \AA$, is compared to the differential broadening of spectral lines with large and small Landé factors in order to produce a relation to estimate the field strengths of magnetic stars with unresolved Zeeman patterns.

Results. The method is shown to be reliable for rotational velocities up to about $50 \mathrm{~km} \mathrm{~s}^{-1}$ for field strengths down to about $5 \mathrm{kG}$.

Conclusions. These results should allow for better constraints to be placed on the mean surface magnetic fields of Ap stars where Zeeman patterns are unresolved.
\end{abstract}

Key words. stars: magnetic field - stars: chemically peculiar

\section{Introduction}

The detections of magnetic fields in stars of the upper main sequence rely on observing the Zeeman effect in spectral lines. This is generally done by either observing polarisation or directly measuring Zeeman splitting in stellar spectral lines. The former furnishes the line-of-sight magnetic field, $\left\langle B_{z}\right\rangle$, whereas the latter gives the surface magnetic field, $\langle B\rangle$.

The mean line-of-sight (or longitudinal) magnetic field is a line intensity weighted average over the visible hemisphere of a star of the magnetic field component directed along the lineof-sight. It is obtained from measuring the separation between the positions of the spectral line profiles in left and right circularly polarised light. Observed variations in $\left\langle B_{z}\right\rangle$ for a given star provide constraints for the magnetic field structure (that is assumed to be symmetric about an axis that is inclined to the rotation axis) such as the mean and maximum/minimum $\left\langle B_{z}\right\rangle$ values. However, Mathys (2004) points out that characterising the actual stellar magnetic field from the longitudinal field alone is not possible due to the strong dependence on the geometry of the observation.

* Based on observations made with the European Southern Observatory telescopes under ESO programmes 60.A-9036(A), 68.D-0254(A), 69.D-0378(A), 074.D-0392(A), 075.C-0234(A), 075.C-0234(B), 076.C-0073(A), 076.D-0169(A), 077.D-0477(A), 079.D-0118(A), 079.C-0170(A), 079.C-0170(B), 080.C-0032(A), 080.C-0032(B), 081.C-0034(A), 081.C-0034(B), 082.C-0218(A), 082.C-0308(A), 089.C-0207(A), 089.D-0383(A), 266.D-5655(A), obtained from the ESO/ST-ECF Science Archive Facility. Based on observations obtained at the Canada-France-Hawaii Telescope (CFHT) which is operated by the National Research Council of Canada, the Institut National des Science de l'Univers of the Centre National de la Recherche Scientifique of France, and the University of Hawaii. Based in part on observations available on the ELODIE archive.
The mean surface magnetic field strength can be measured from resolved Zeeman splitting in a spectral line, providing a line intensity weighted average over the visible stellar hemisphere of the modulus of the magnetic field. Zeeman splitting is difficult to measure in spectral lines because even small rotational velocities can mask this signature. However, if the rotational velocity broadening is sufficiently small compared to the magnetic field splitting (usually the field strength in $\mathrm{kG}$ should be larger than the rotational velocity in $\mathrm{km} \mathrm{s}^{-1}$ ), it is possible to measure $\langle B\rangle$ directly from observed Zeeman patterns in a stellar spectrum (see Landstreet \& Mathys 2000). This provides further constraints on the magnetic field configuration (maximum and minimum $\langle B\rangle$ values) allowing for a more detailed picture of the overall field structure (see Landstreet 2009).

The magnetic chemically peculiar A- type stars (Ap) host the largest magnetic fields of stars on the main sequence, with $\left\langle B_{z}\right\rangle$ ranging in strength from hundreds to thousands of Gauss. Generally, sinusoidal variations in $\left\langle B_{z}\right\rangle$ are observed over the rotational period of the star. The simplest model to describe these variations is an oblique rotator that consists of the angles $i$ and $\beta$, describing the inclinations of the line-of-sight and magnetic field axes to the rotation axis, respectively (see Landstreet 1970, for a clear explanation of rigid rotator model). Therefore, to completely specify the magnetic field geometry requires knowledge of three parameters $i, \beta$ and the polar field strength $B_{\mathrm{d}}$. As such, variations in $\left\langle B_{z}\right\rangle$ alone cannot supply sufficient constraints to specify a unique geometry; however, with the addition of $\langle B\rangle$, not only can we specify all three parameters, but also additional field components such as a magnetic quadrupole aligned with a magnetic dipole (see Landstreet 2009, for a more detailed description). Thus, $\langle B\rangle$ is integral in providing the overall field structure, but most often times Zeeman patterns are undetectable because the star rotates too quickly. In such cases, stars are diagnosed as being magnetic only from measurements of $\left\langle B_{z}\right\rangle$. 
Mathys (1995) combats the problem of unresolved Zeeman patterns in magnetic stars by introducing the mean quadratic field, $B_{\mathrm{mq}}$. The mean quadratic field is derived from the measure of the second order moments of the Stokes $I$ parameter. It characterises the widths of spectral lines about their centres due to magnetic broadening. In particular, the mean quadratic field is the square root of the sum of the squares of the mean surface magnetic field and the line-of-sight magnetic field: $B_{\mathrm{mq}}=$ $\left(\left\langle B^{2}\right\rangle+\left\langle B_{z}^{2}\right\rangle\right)^{0.5}$. The lower limit to the success of this method was found to be about $5 \mathrm{kG}$.

Preston (1971) suggests a simpler method in characterising the mean surface fields of rotationally broadened stars, which also exploits the fact that magnetic fields can broaden spectral lines such that the broadening is significant compared to the dominant rotational broadening. Preston measured the mean surface fields for seven stars with resolved Zeeman patterns and for these same stars compared the widths of spectral lines that are strongly affected by the local magnetic field to ones that are not, finding a linear correlation between spectral line widths and magnetic field strength. Recently, this relationship was used by Bailey et al. (2012) to constrain $\langle B\rangle$ in a star (HD 133880) with $v \sin i$ larger than $100 \mathrm{~km} \mathrm{~s}^{-1}$.

This paper aims to improve upon the relation of Preston (1971) with the goal of extending this work to be used on rapidly rotating magnetic Ap stars to better constrain their magnetic fields without having to rely solely on line-of-sight magnetic measurements from circular polarisation. This is achieved by utilising an extensive collection of high dispersion spectra of magnetic stars with resolved Zeeman splitting from the ESO and CFHT archives: a total of 18 stars and 102 spectra. This study also benefits from the much higher signal-to-noise ratio (SNR) and increased linearity of today's instruments. The following section discusses the observations. Sections 3 and 4 describe the methods and results, respectively. Section 5 tests the method by comparing to synthetic spectra and Sect. 6 outlines the conclusions from this work.

\section{Observations}

The majority of data used for this study were obtained from the European Southern Observatory (ESO) and Canada-FranceHawaii Telescope (CFHT) archives (using the UVES, HARPS and ESPaDOnS instruments), with selected spectra also taken from the ELODIE archive, for a total of 102 high resolution spectra for 18 magnetic stars with resolved Zeeman patterns. Table 1 lists the stars used for this study. Below I describe each instrument in brief.

ELODIE was a spectrograph located at Observatoire de Haute Provence. It covered a spectral range from about 3900 to $6800 \AA$ with a resolving power of $R \simeq 42000$. All the ELODIE spectra utilised in this study have SNR of about 100 .

ESPaDOnS, a cross-dispersed echelle spectropolarimeter, is located at the CFHT. The instrument covers a spectral range from 3690 to $10481 \AA$ with a resolving power of $R \simeq 65000$ (in polarimetric mode). The available spectra have SNR above about 200.

Located at the ESO La Silla $3.6 \mathrm{~m}$ telescope, HARPS is a cross-dispersed echelle spectrograph covering a spectral range of 3780-6910 $\AA$ with $R \simeq 115000$. The SNR of the available spectra were also of order 200.

UVES is a cross-dispersed optical spectrograph that is located at ESO's Paranal Observatory. It has both a blue and red
Table 1. Star designations, instrument (with the number of spectra indicated in parentheses), spectral resolution, and spectral range are listed for the stars used in this study.

\begin{tabular}{lrrr}
\hline \hline Star & Instrument & $R$ & $\lambda(\AA)$ \\
\hline HD 2453 & ELODIE (1) & 42000 & $3900-6800$ \\
& UVES (1) & 80000 & $3282-4563$ \\
HD 12288 & ELODIE (1) & 110000 & $57000-9463$ \\
HD 51684 & UVES (2) & 80000 & $3900-6800$ \\
& & 110000 & $4727-6563$ \\
HD 65339 & ESPaDOnS (1) & 65000 & $3690-10481$ \\
HD 93507 & HARPS (12) & 115000 & $3780-6910$ \\
HD 94660 & ESPaDOnS (1) & 65000 & $3690-10481$ \\
& HARPS (2) & 115000 & $3780-6910$ \\
HD 116114 & HARPS (14) & 115000 & $3780-6910$ \\
HD 116458 & HARPS (10) & 115000 & $3780-6910$ \\
HD 126515 & UVES (1) & 80000 & $3731-4999$ \\
& & 110000 & $4726-6835$ \\
HD 137909 & ESPARPS (10) & 115000 & $3780-6910$ \\
& HARPS (1) & 65000 & $3690-10481$ \\
HD 142070 & HARPS (6) & 115000 & $3780-6910$ \\
HD 144897 & HARPS (5) & 115000 & $3780-6910$ \\
HD 166473 & UVES (1) & 80000 & $3780-6910$ \\
& & 110000 & $4731-4999$ \\
& HARPS (7) & 115000 & $3780-69310$ \\
HD 187474 & UVES (1) & 80000 & $3731-4999$ \\
& & 110000 & $4727-6835$ \\
HD 188041 & HARPS (2) & 115000 & $3780-6910$ \\
HD 192678 & ELODIE (1) & 42000 & $3900-6800$ \\
HD 208217 & HARPS (9) & 115000 & $3780-6910$ \\
HD 318107 & ESPaDOnS (2) & 65000 & $3690-10481$ \\
& HARPS (7) & 115000 & $3780-6910$ \\
\hline & & &
\end{tabular}

arm that offers different spectral resolutions and range coverage. The blue arm, with $R \simeq 80000$, covers a spectral range from about 3100 to $4900 \AA$, whereas the red arm $(R \simeq 110000)$ can range from about 4800 to $10200 \AA$. The SNR of the available spectra were above about 300 .

\section{Measurements}

I endeavour to obtain an estimate for $\langle B\rangle$ from comparing the widths of two spectral lines that have large and small Landé factors $z$ (i.e. lines that are strongly affected by the local magnetic field to ones that are not). To achieve this, I adapt the measurement technique of Preston (1971) which is summarised below.

\subsection{The K-parameter}

If one considers the $\sigma-\sigma$ separation of Zeeman split components, it is straightforward to show that the width of a line broadened by the magnetic field can be written as const. $\times \lambda^{2} z$. Preston (1971) defines this constant as the parameter $K$, which is proportional to the strength of the magnetic field, $\langle B\rangle$. Using the same notation as Preston (1971), if it is further assumed that the instrumental $\left(w_{\mathrm{I}}\right)$ and magnetic broadening profiles can be added in quadrature then

$w_{\mathrm{L}}^{2}=w_{\mathrm{I}}^{2}+K^{2}\left\langle\lambda^{4} z^{2}\right\rangle_{\mathrm{L}}$,

$w_{\mathrm{S}}^{2}=w_{\mathrm{I}}^{2}+K^{2}\left\langle\lambda^{4} z^{2}\right\rangle_{\mathrm{S}}$, 
Table 2. Fe II lines used to measure widths.

\begin{tabular}{rrrr}
\hline \hline \multicolumn{2}{c}{ Large $z$} & \multicolumn{2}{c}{ Small $z$} \\
\hline$\lambda(\AA)$ & $z$ & $\lambda(\AA)$ & $z$ \\
\hline 4271.404 & 1.33 & 4491.400 & 0.40 \\
4303.176 & 1.43 & 4508.280 & 0.50 \\
4520.220 & 1.34 & & \\
\hline
\end{tabular}

Notes. Listed are the rest wavelengths and Landé $z$ factors.

where $w_{\mathrm{L}}$ and $w_{\mathrm{S}}$ are the widths for lines with large and small $z$ values, respectively. Combining Eqs. (1a) and (1b), the $K$ parameter can be written as

$K=\left(\frac{w_{\mathrm{L}}^{2}-w_{\mathrm{S}}^{2}}{\left\langle\lambda^{4} z^{2}\right\rangle_{\mathrm{L}}-\left\langle\lambda^{4} z^{2}\right\rangle_{\mathrm{S}}}\right)^{1 / 2}$.

Note that for this relation, both the widths and wavelengths are measured in centimetres meaning that $K$ is the wavenumber (i.e. units of $\mathrm{cm}^{-1}$ ), which are the same units used by Preston (1971).

\subsection{Technique}

For each star listed in Table 1, the mean magnetic surface fields were measured from the observed splitting in the Fe II 6149 line. In addition to this, for each star the widths of up to five spectral lines in Table 2 were measured. To obtain accurate and consistent measurements, I had to consider that the lines with large $z$ values could have multiple Zeeman components which would make fitting a simple Gaussian infeasible. Therefore, for each line the full width at half-depth (FWHD) was measured. The widths of each line were then used to measure the $K$-parameter from Eq. (2) with the necessary atomic data collected from the Vienna Atomic Line Database (VALD; Kupka et al. 2000; Ryabchikova et al. 1997; Piskunov et al. 1995; Kupka et al. 1999). For each spectrum, an average $K$ value was found from all possible combinations of lines with large and small $z$ values with an estimated uncertainty that corresponds to the largest spread in $K$ values from different sets of lines. The uncertainties in $\langle B\rangle$ measured from Fe II 6149 were estimated by considering the scatter about the mean fit of multiple individual measurements for each spectrum. Table 3 lists these average $K$ values and $\langle B\rangle$ from Fe II 6149, with associated uncertainties, for each star. For stars (or multiple spectra for the same star) which exhibit little variation between $\langle B\rangle$ measurements, only mean values for $\langle B\rangle$ and $K$ are recorded in Table 3 . These are denoted by an asterisk.

\section{Results}

Figure 1 plots the relation between $K$ and $\langle B\rangle$, showing a clear linear relation. The best-fit line (solid black) of all the data suggests that $\langle B\rangle(\mathrm{kG})=0.068+8.05 K$. However, for a star without a magnetic field, in which $K$ is zero, the measured field from the relation should also be zero. Therefore, also shown is the linear fit with the $y$-intercept forced through the origin (black dashed line). Both lines fit the data equally well; therefore, since the fitted relation should pass through the origin, the latter relation with a zero constant term is adopted with

$\langle B\rangle(\mathrm{kG})=8.11 K$.

This equation is used to calculate the surface magnetic field strength in Table 3.
Table 3. $K$ value, $\langle B\rangle$ measurements and estimated surface field strengths from Eq. (3), with associated uncertainties, for each star.

\begin{tabular}{|c|c|c|c|}
\hline \multirow[t]{2}{*}{ Star } & \multirow[t]{2}{*}{$K\left(\mathrm{~cm}^{-1}\right)$} & \multicolumn{2}{|c|}{$\langle B\rangle(\mathrm{kG})$} \\
\hline & & Fe II 6149 & Eq. (3) \\
\hline HD 2453 & $0.41 \pm 0.09 *$ & $3.50 \pm 0.42 *$ & $3.33 \pm 0.73$ \\
\hline HD 12288 & $1.01 \pm 0.08$ & $8.36 \pm 0.61$ & $8.19 \pm 0.65$ \\
\hline HD 51684 & $0.87 \pm 0.10 *$ & $6.18 \pm 0.12 *$ & $7.06 \pm 0.81$ \\
\hline HD 65339 & $0.96 \pm 0.07$ & $8.52 \pm 0.42$ & $7.79 \pm 0.57$ \\
\hline \multirow[t]{4}{*}{ HD 93507} & $0.93 \pm 0.08 *$ & $7.04 \pm 0.29 *$ & $7.54 \pm 0.65$ \\
\hline & $1.00 \pm 0.06^{*}$ & $7.42 \pm 0.39 *$ & $8.11 \pm 0.49$ \\
\hline & $1.03 \pm 0.10^{*}$ & $7.71 \pm 0.35^{*}$ & $8.35 \pm 0.81$ \\
\hline & $1.09 \pm 0.05^{*}$ & $8.70 \pm 0.22 *$ & $8.84 \pm 0.41$ \\
\hline HD 94660 & $0.80 \pm 0.05^{*}$ & $6.26 \pm 0.21 *$ & $6.49 \pm 0.41$ \\
\hline HD 116114 & $0.77 \pm 0.07 *$ & $6.13 \pm 0.36^{*}$ & $6.24 \pm 0.57$ \\
\hline HD 116458 & $0.58 \pm 0.06^{*}$ & $4.63 \pm 0.23^{*}$ & $4.70 \pm 0.49$ \\
\hline \multirow[t]{8}{*}{ HD 126515} & $1.14 \pm 0.10 *$ & $9.31 \pm 0.21^{*}$ & $9.25 \pm 0.81$ \\
\hline & $1.19 \pm 0.04$ & $9.89 \pm 0.17$ & $9.65 \pm 0.32$ \\
\hline & $1.26 \pm 0.05$ & $10.25 \pm 0.35$ & $10.22 \pm 0.41$ \\
\hline & $1.30 \pm 0.07$ & $10.53 \pm 0.35$ & $10.54 \pm 0.57$ \\
\hline & $1.32 \pm 0.06$ & $10.92 \pm 0.19$ & $10.71 \pm 0.49$ \\
\hline & $1.45 \pm 0.10$ & $11.40 \pm 0.57$ & $11.76 \pm 0.81$ \\
\hline & $1.67 \pm 0.08$ & $13.02 \pm 0.33$ & $13.54 \pm 0.65$ \\
\hline & $1.86 \pm 0.08^{*}$ & $14.82 \pm 0.39 *$ & $15.08 \pm 0.65$ \\
\hline HD 137909 & $0.61 \pm 0.09 *$ & $5.24 \pm 0.34 *$ & $4.93 \pm 0.73$ \\
\hline HD 142070 & $0.54 \pm 0.06^{*}$ & $4.54 \pm 0.40 *$ & $4.34 \pm 0.49$ \\
\hline \multirow{5}{*}{ HD 144897} & $1.07 \pm 0.06$ & $8.45 \pm 0.11$ & $8.68 \pm 0.49$ \\
\hline & $1.07 \pm 0.07$ & $8.62 \pm 0.16$ & $8.68 \pm 0.57$ \\
\hline & $1.09 \pm 0.06$ & $8.80 \pm 0.27$ & $8.84 \pm 0.49$ \\
\hline & $1.17 \pm 0.09$ & $9.45 \pm 0.13$ & $9.49 \pm 0.73$ \\
\hline & $1.17 \pm 0.07$ & $9.60 \pm 0.21$ & $9.49 \pm 0.57$ \\
\hline \multirow[t]{2}{*}{ HD 166473} & $0.75 \pm 0.08^{*}$ & $6.11 \pm 0.32 *$ & $6.08 \pm 0.65$ \\
\hline & $1.02 \pm 0.06$ & $8.68 \pm 0.11$ & $8.27 \pm 0.49$ \\
\hline HD 187474 & $0.70 \pm 0.06$ & $5.75 \pm 0.10$ & $5.65 \pm 0.49$ \\
\hline HD 188041 & $0.38 \pm 0.03^{*}$ & $3.52 \pm 0.39 *$ & $3.08 \pm 0.24$ \\
\hline HD 192678 & $0.47 \pm 0.04$ & $4.43 \pm 0.30$ & $3.83 \pm 0.33$ \\
\hline \multirow[t]{4}{*}{ HD 208217} & $0.94 \pm 0.07$ & $7.56 \pm 0.38$ & $7.66 \pm 0.57$ \\
\hline & $0.96 \pm 0.07$ & $7.91 \pm 0.40$ & $7.79 \pm 0.57$ \\
\hline & $1.08 \pm 0.09 *$ & $8.41 \pm 0.35^{*}$ & $8.76 \pm 0.73$ \\
\hline & $1.15 \pm 0.10 *$ & $9.16 \pm 0.39 *$ & $9.33 \pm 0.81$ \\
\hline \multirow[t]{3}{*}{ HD 318107} & $1.64 \pm 0.06$ & $13.57 \pm 0.26$ & $13.30 \pm 0.48$ \\
\hline & $1.68 \pm 0.07 *$ & $13.84 \pm 0.32 *$ & $13.62 \pm 0.57$ \\
\hline & $1.94 \pm 0.06$ & $16.11 \pm 0.49$ & $15.73 \pm 0.49$ \\
\hline
\end{tabular}

Notes. For stars with spectra that exhibit little $\langle B\rangle$ variability, an average value of multiple spectra is shown. ${ }^{(*)}$ Denotes an average of multiple spectra.

\subsection{Effects of rotational broadening}

The goal of this work is to measure $K$ values for stars with larger $v \sin i\left(\gtrsim 20 \mathrm{~km} \mathrm{~s}^{-1}\right)$ in order to estimate $\langle B\rangle$. Therefore, it is important to ascertain how feasible it is to measure magnetic broadening in spectral lines as rotational broadening increases. To investigate this, it is necessary to compare spectra with and without the effects of a magnetic field. ZEEMAN is a FORTRAN program that synthesises stellar spectra and is capable of including the effects of a magnetic field (detailed descriptions of ZEEMAN are provided by Landstreet 1988; Wade et al. 2001).

ZEEMAN was used to create pairs of synthetic spectra for $\mathrm{Fe}$ II 4520 (with and without the presence of a magnetic field) for incrementally increasing values of $v \sin i$. For this comparison, a field strength of $15 \mathrm{kG}$ was used. The results are shown in Fig. 2. One can see how the Zeeman splitting in the Fe II 4520 line slowly disappears as $v \sin i$ increases, but magnetic broadening still remains. Above about $50 \mathrm{~km} \mathrm{~s}^{-1}$, the magnetic broadening is only marginally larger than the rotational broadening. Table 4 


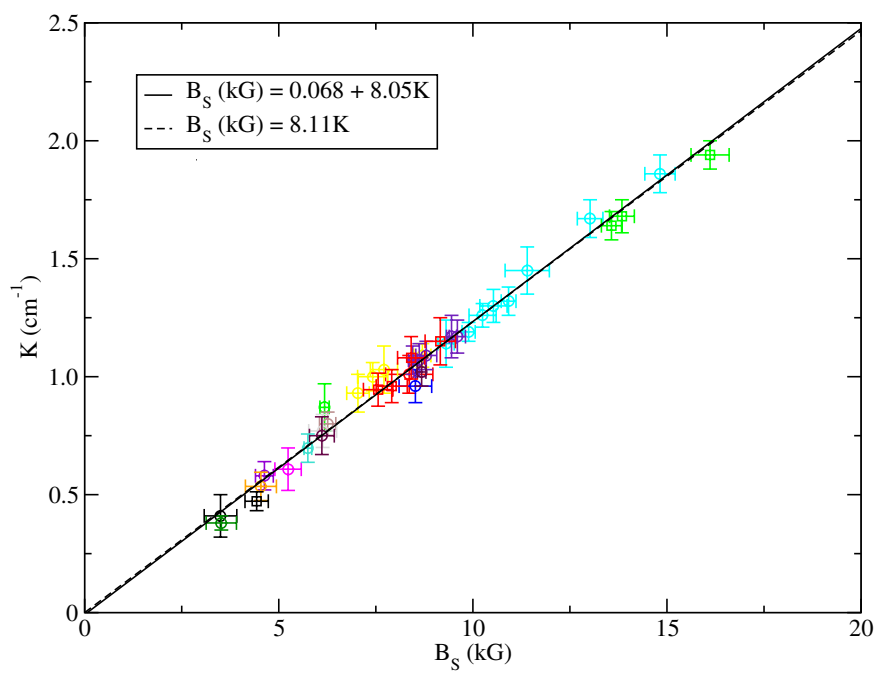

Fig. 1. Measured $K$-parameters versus the surface field strengths (as measured from observed splitting in Fe II 6149) from the values in Table 3. Each colour and/or shape indicates measurements from an individual star. The solid line is the best-fit line of all the data and the dotted black line is the best-fit when forcing the $y$-intercept through the origin.

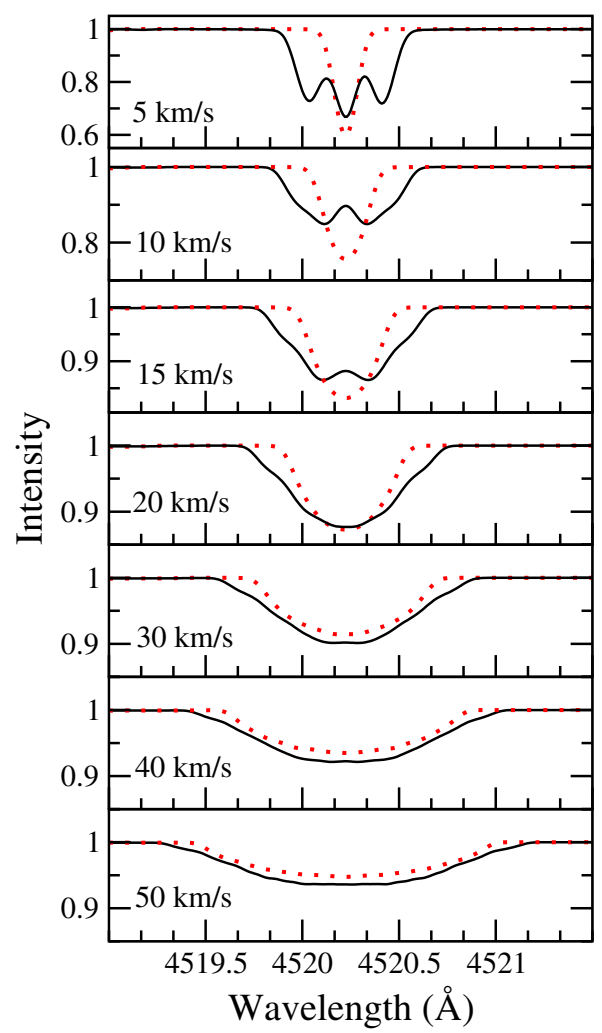

Fig. 2. Comparisons between the Fe II 4520 line for a $15 \mathrm{kG}$ field (black lines) and no magnetic field (red dots) for increasing values of $v \sin i$.

lists the FWHD for the lines in Fig. 2, with typical uncertainties of order $0.005 \AA$, as determined from the scatter of multiple measurements. Even for values of $v \sin i$ of about $50 \mathrm{~km} \mathrm{~s}^{-1}$, there is still a measurable difference between the rotationally broadened and magnetically and rotationally broadened spectral line of Fe II at $4520 \AA$; however, this difference is marginal, suggesting that it may be difficult to obtain an accurate measure of the field above about $50 \mathrm{~km} \mathrm{~s}^{-1}$. Note that Fe II 4520 has a Landé

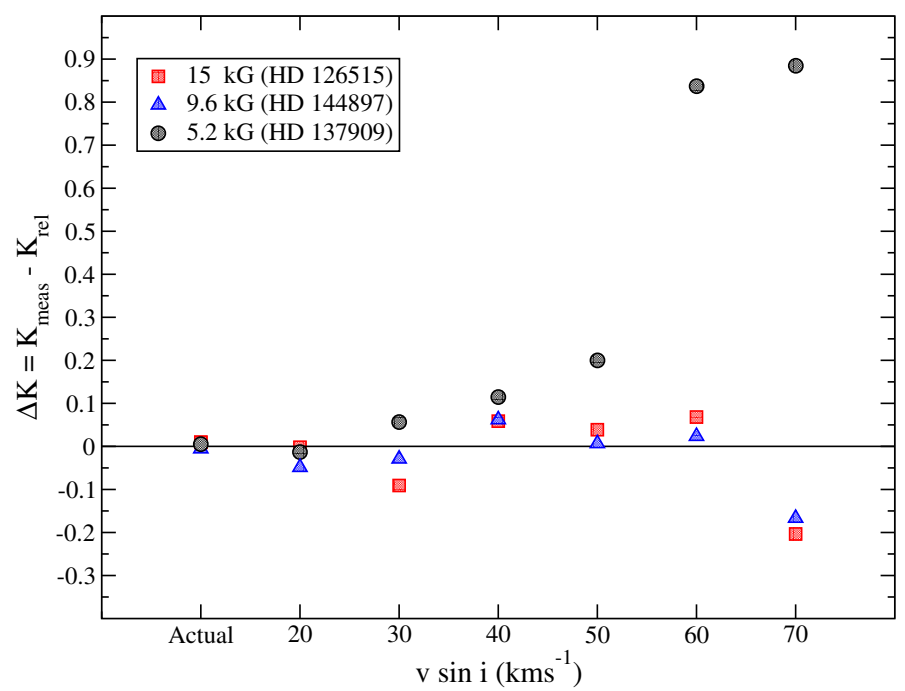

Fig. 3. Difference between the measured and predicted $K$-parameters for three different field strengths at various rotation rates. The actual measurement, when deriving the relation of Eq. (2) is also shown. The black circles are for HD 137909 with $\langle B\rangle=5.2 \mathrm{kG}$, the blue triangles are for HD 144897 with $\langle B\rangle=9.6 \mathrm{kG}$ and the red square are for HD 126515 with $\langle B\rangle=15 \mathrm{kG}$.

Table 4. Measured full width at half depth (FWHD) for Fe II 4520 at multiple values of $v \sin i$ for a field strength of $15 \mathrm{kG}$.

\begin{tabular}{lrrr}
\hline \hline \multirow{2}{*}{$\begin{array}{l}\text { sin } i \\
\left(\mathrm{~km} \mathrm{~s}^{-1}\right)\end{array}$} & No $B$ & $B W H D(\AA)$ \\
\hline 5 & 0.135 & 0.513 & 0.378 \\
10 & 0.234 & 0.568 & 0.334 \\
15 & 0.350 & 0.580 & 0.230 \\
20 & 0.472 & 0.614 & 0.142 \\
30 & 0.720 & 0.784 & 0.064 \\
40 & 0.947 & 0.981 & 0.034 \\
50 & 1.197 & 1.207 & 0.010 \\
\hline
\end{tabular}

Notes. Shown are the results without and with a magnetic field and the difference between the two FWHD. The measurements have typical uncertainties of order $0.005 \AA$.

factor $z=1.34$ and that the choice of a different spectral line with a larger $z$ value would increase the difference, perhaps making it plausible to measure the field in stars with even larger rotation rates.

\subsection{Artificial broadening of real spectra}

The preceding section suggests that reliably measuring magnetic broadening in a spectral line may be difficult above a rotational velocity of about $50 \mathrm{~km} \mathrm{~s}^{-1}$. Therefore, in order to ascertain the reproducibility of the calculated $K$ values at large $v \sin i$, the spectra of three separate stars with field strengths around 5, 10 and $15 \mathrm{kG}$ (HD 137909, HD 144897 and HD 126515, respectively) were convolved with the rotational broadening function (e.g. Unsold 1955). The spectra were broadened up to $70 \mathrm{~km} \mathrm{~s}^{-1}$ in intervals of $10 \mathrm{~km} \mathrm{~s}^{-1}$. For each spectrum, the $K$ values were measured in the same manner as described in Sect. 3. Figure 3 shows the difference between the measured $K$ values and the $K$ value predicted by Eq. (2) plotted against $v \sin i$ for the rotationally broadened spectra. Also shown is the actual measurement obtained when deriving the relation.

At both 10 and $15 \mathrm{kG}$, the $K$ value is consistently reproduced at all $v \sin i$, with a slightly larger disparity at $70 \mathrm{~km} \mathrm{~s}^{-1}$ 
of about $0.2 \mathrm{~cm}^{-1}$. Above about $50 \mathrm{~km} \mathrm{~s}^{-1}$, the $K$ value cannot be reliably measured for a field of about $5 \mathrm{kG}$. Apparently the marginal difference in the FWHD at larger $v \sin i$ shown in Fig. 2 and Table 4 is sufficient to reproduce the measured $K$ value from magnetic broadening for a field above about $10 \mathrm{kG}$ up to about 60 or $70 \mathrm{~km} \mathrm{~s}^{-1}$. It is important to note that although Fig. 3 suggests that larger magnetic fields follow the linear relationship of Eq. (3) above $v \sin i$ of about $50 \mathrm{~km} \mathrm{~s}^{-1}$, no a priori knowledge of the field strength will exist when a measurement is made for an actual star. Therefore, for the $v \sin i$ 's considered, the method becomes unreliable above about $50 \mathrm{~km} \mathrm{~s}^{-1}$ for $\langle B\rangle$ less than or equal to about $15 \mathrm{kG}$ because it is impossible to know whether the measurement yields a reliable $\langle B\rangle$ value or not.

\section{Comparison to synthetic spectra}

The reproducibility of the $K$ value at large $v \sin i$ 's suggests that Eq. (3) may be reliably used to estimate the field strengths of magnetic stars that are fast rotators. In Sect. 4.2, observed spectra were artificially broadened to determine how accurately the $K$ parameter can be reproduced with increasing $v \sin i$. However, the question still remains of how accurate the relation is at measuring the surface field of a star for which the field strength is known precisely. To test this, I used the program ZEEMAN (see Sect. 4). In addition to including the effects of magnetic fields, ZEEMAN also allows as input the specification of a simple magnetic field geometry that is a simple co-axial multipole expansion consisting of dipole, quadrupole and octupole components with the angles between the line-of-sight and rotation axis $(i)$ and the magnetic field and rotation axis $(\beta)$ specified. As output, ZEEMAN also provides the precise surface field strength. Therefore, it is possible to compare the estimated field strength from Eq. (3) to spectra for which the exact field value is known.

Only Fe lines are used to measure $K$ and since the magnetic stars in this study are most certainly Ap in nature, the abundance of $\mathrm{Fe}$ was set to a value that is 0.5 dex above the solar ratio. To test the effects of varying geometries, synthetic spectra for $v \sin i$ 's up to $70 \mathrm{~km} \mathrm{~s}^{-1}$ were produced for simple dipolar field strengths of 5, 10, 15 and $20 \mathrm{kG}$ with $i=0,45$ and 90 degrees. To test the applicability of this method of magnetic field determination for increasing $v \sin i$, a geometry where the magnetic field axis is perpendicular to the rotation axis (i.e. $\beta=90^{\circ}$ ) was favoured, because the angle between the rotation and magnetic field axes are statistically larger in faster rotators (Landstreet \& Mathys 2000). It is recognised that the surface field measured depends not only on the mean value $\langle B\rangle$, but also, to some extent, on the organisation of the field. However, the precision of this method is insufficient to detect these types of changes. As such, the phase dependence in measuring $\langle B\rangle$ with this method is not tested and all computations are performed at the same phase, ensuring that the line-of-sight lies in the plane defined by the rotation and magnetic field axes.

Figure 4 shows the percent difference between the measured $\langle B\rangle$ values from Eq. (3) to the actual $\langle B\rangle$ value from the synthetic spectra (as reported by ZEEMAN) plotted against $v \sin i$. The approximate field strength is shown in each panel. Note that no significant differences were found between the three different geometries and therefore only the results for the pole-on geometry are shown. For fields greater than about $12 \mathrm{kG}$, the predicted field strength from Eq. (3) accurately predicts the surface field strength to within $6 \%$ at all $v \sin i$ considered. This is also true up to a rotation of $50 \mathrm{~km} \mathrm{~s}^{-1}$ for a field of about $8 \mathrm{kG}$. Above this $v \sin i$, the measured value is within $25 \%$ for an $8 \mathrm{kG}$ field. At the lower field strength of $4 \mathrm{kG}$, the field is predicted

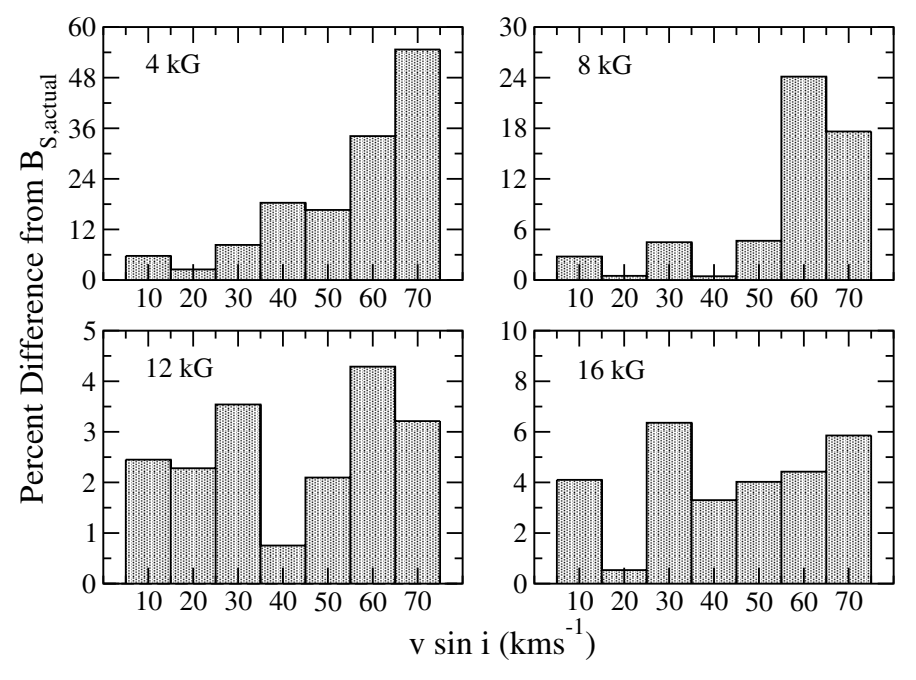

Fig. 4. Percent differences between the measured $\langle B\rangle$ values from Eq. (3) to the actual $\langle B\rangle$ value from the synthetic spectra (as reported by ZEEMAN) plotted against $v \sin i$. The approximate field strength is shown in each panel. Note that the $y$-axis is different for each panel.

within $20 \%$ at or below a rotation of $50 \mathrm{~km} \mathrm{~s}^{-1}$ and quickly diverges at higher $v \sin i$ values. These results can be understood by considering that at lower field values it becomes increasingly difficult to discern between rotational and magnetic broadening in a spectral line whereas for larger field values the magnetic broadening is still significant compared to the rotational broadening (see Fig. 2).

\section{Conclusions}

In this paper, a relation to estimate the surface magnetic field $\langle B\rangle$ for stars with large $v \sin i$ (above about $10 \mathrm{~km} \mathrm{~s}^{-1}$ ) was derived. This was done by extending the work of Preston (1971) to include 102 total spectra for 18 magnetic stars with resolved Zeeman splitting. For each spectrum, the surface field strengths were measured from the observed splitting in Fe II 6149 and the widths of spectral lines with large and small Landé factors were measured to determine the $K$ parameter (a constant that is proportional to the magnetic field strength). The derived relation is presented in Eq. (3).

The $K$ parameter was shown to be reliably reproduced at increasingly large $v \sin i$ when the spectra were rotationally broadened. For lower field values (around $5 \mathrm{kG}$ ), the $K$ parameter is not accurately reproduced above about $50 \mathrm{~km} \mathrm{~s}^{-1}$.

The relation of Eq. (3) was also compared to synthetic spectra for which the surface fields were known precisely. It was found that for $\langle B\rangle$ greater than about $8 \mathrm{kG}$ the field was accurately predicted to within $6 \%$ of the actual value. Below about $10 \mathrm{kG}$, the ability to accurately measure the magnetic field above $v \sin i \simeq 50 \mathrm{~km} \mathrm{~s}^{-1}$ diminishes to within $25 \%$ and is completely unreliable for surface fields of order 8 and $4 \mathrm{kG}$, respectively. Below about $50 \mathrm{~km} \mathrm{~s}^{-1}$, a $4 \mathrm{kG}$ field can be measured to within about $20 \%$ with increased precision as $v \sin i$ decreases. However, since no knowledge of the surface field strength exists prior to measuring the field from this technique, the method is only useful up to $v \sin i$ of about $50 \mathrm{~km} \mathrm{~s}^{-1}$ and for magnetic field strengths down to about $5 \mathrm{kG}$.

These results are very encouraging and demonstrate that this simple method can reliably estimate the surface field strengths of magnetic stars with large $v \sin i$ (up to about $50 \mathrm{~km} \mathrm{~s}^{-1}$ ). Thus, this technique will allow constraints to be placed on the 
mean surface field variations of magnetic stars for which only line-of-sight measurements were previously possible.

Acknowledgements. J.D.B. acknowledges support from the University of Leeds and thanks the referee Gautier Mathys for helpful comments. J.D.B. is also grateful to John D. Landstreet for many useful discussions and graciously reviewing the manuscript. Lastly, J.D.B. thanks Drs Nicole Bailey and Andy Pon for many helpful hints with IDL.

\section{References}

Bailey, J. D., Grunhut, J., Shultz, M., et al. 2012, MNRAS, 423, 328

Kupka, F., Piskunov, N. E., Ryabchikova, T. A., Stempels, N. C., \& Weiss, W. W. 1999, A\&A, 138, 119
Kupka, F. G., Ryabchikova, T. A., Piskunov, N. E., Stempels, H. C., \& Weiss, W. W. 2000, Baltic Astron., 9, 590

Landstreet, J. D. 1970, ApJ, 159, 1001

Landstreet, J. D. 1988, ApJ, 326, 967

Landstreet, J. D. 2009, in EAS Pub. Ser. 39, eds. C. Neiner, \& J.-P. Zahn, 1

Landstreet, J. D., \& Mathys, G. 2000, A\&A, 359, 213

Mathys, G. 1995, A\&A, 293, 746

Mathys, G. 2004, in The A-Star Puzzle, eds. J. Zverko, J. Ziznovsky, S. J. Adelman, \& W. W. Weiss, IAU Symp., 224, 225

Piskunov, N. E., Kupka, F., Ryabchikova, T. A., Weiss, W. W., \& Jeffery, C. S. 1995, A\&A, 112, 525

Preston, G. W. 1971, ApJ, 164, 309

Ryabchikova, T. A., Piskunov, N. E., Kupka, F., \& Weiss, W. W. 1997, Baltic Astron., 6, 244

Unsold, A. 1955, Physik der Sternatmospharen (Berlin: Springer)

Wade, G. A., Bagnulo, S., Kochukhov, O., et al. 2001, A\&A, 374, 265 